\title{
FOLDS AND CUSPS IN BANACH SPACES WITH APPLICATIONS TO NONLINEAR PARTIAL DIFFERENTIAL EQUATIONS. II
}

\author{
M. S. BERGER, P. T. CHURCH AND J. G. TIMOURIAN
}

\begin{abstract}
Earlier the authors have given abstract properties characterizing the fold and cusp maps on Banach spaces, and these results are applied here to the study of specific nonlinear elliptic boundary value problems. Functional analysis methods are used, specifically, weak solutions in Sobolev spaces. One problem studied is the inhomogeneous nonlinear Dirichlet problem$$
\Delta u+\lambda u-u^{3}=g \quad \text { on } \Omega, \quad u \mid \partial \Omega=0,
$$

where $\Omega \subset \mathbf{R}^{n}(n \leq 4)$ is a bounded domain. Another is a nonlinear elliptic system, the von Kármán equations for the buckling of a thin planar elastic plate when compressive forces are applied to its edge.
\end{abstract}

0. Introduction. This paper continues the research described in [BCT-2] by applying the abstract characterizations of Banach space folds and cusps given there to specific nonlinear elliptic boundary value problems. Our functional analysis approach is sufficiently general that it applies to certain nonlinear elliptic equations, both second and higher order, as well as a nonlinear elliptic system (the von Kármán equations).

For simplicity we have chosen the Hilbert space, weak solution formulation of elliptic boundary value problems in Sobolev space. Occasionally this imposes artificial restrictions on the problem studied, and we shall mention these as they occur. We begin by considering the weak formulation of a specific inhomogeneous nonlinear Dirichlet problem:

$$
\Delta u+\lambda u-u^{3}=g \quad \text { on } \Omega, \quad u \mid \partial \Omega=0
$$

where $\Omega \subset \mathbf{R}^{n}(n \leq 4)$ is a bounded domain. Let $H$ be the Sobolev space $W_{0}^{1,2}(\Omega)$, and define

$$
A: H \times \mathbf{R} \rightarrow H \times \mathbf{R}, \quad(u, \lambda) \rightarrow\left(A_{\lambda}(u), \lambda\right)
$$

by

$$
\left\langle A_{\lambda}(u), \varphi\right\rangle_{H}=\int_{\Omega}\left[\nabla u \nabla \varphi-\lambda u \varphi+u^{3} \varphi\right]
$$

Received by the editors March 23, 1987.

1980 Mathematics Subject Classification (1985 Revision). Primary 35J65, 58C27; Secondary $47 \mathrm{H} 15$.

Key words and phrases. Nonlinear partial differential equations or systems, elliptic boundary value problem, nonlinear Dirichlet problem, fold map, cusp map, von Kármán equations, bifurcation, singularity theory in infinite dimensions. 


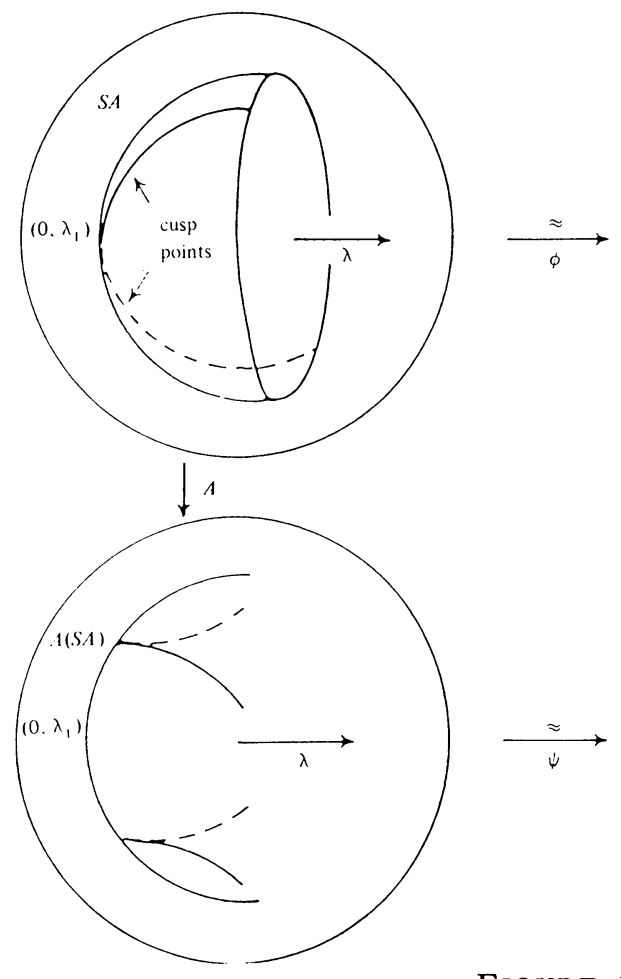

FIGURE 1

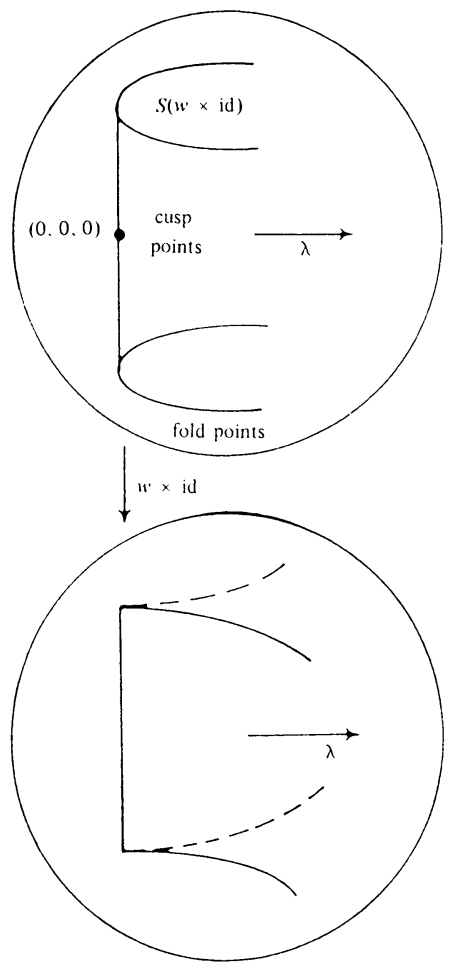

and $\tilde{g} \in H$ by $\langle\tilde{g}, \varphi\rangle_{H}=-\int_{\Omega} g \varphi$ for all test functions $\varphi \in C_{0}^{\infty}(\Omega)$; thus the weak solutions of $(0.1)$ are the solutions $u$ of $A_{\lambda}(u)=\tilde{g}$, i.e. the point inverses of the $\operatorname{map} A$.

Actually the work is generalized beyond equation (0.1) to certain nonlinear operators $A_{\lambda}: H \rightarrow H$ defined on some Hilbert space (1.2), e.g. certain equations (1.4) in the form $\Delta u+\lambda u-f(u)=g$ with $u \mid \partial \Omega=0$, or the operator $A_{\lambda}$ defined by von Kármán equations (4.1). Many of the proofs are given in this more general context.

In this paper we investigate the structure of the real analytic map $A: A$ is a diffeomorphism for $\lambda<\lambda_{1}$ (2.3), where $0<\lambda_{1}<\lambda_{2} \leq \cdots$ are the eigenvalues of minus the Laplacian $-\Delta$ on $\Omega$ with null boundary conditions, and $A_{\lambda_{1}}$ is a homeomorphism (2.10). Thus for $\lambda \leq \lambda_{1}$ solutions of equation (0.1) exist and are unique.

For $\lambda>\lambda_{1}$ the situation is considerably more complicated, and we characterize the bifurcation for $\lambda$ near $\lambda_{1}$ and $g$ near 0 as follows: there are (3.8) a connected open neighborhood $V$ of $\left(0, \lambda_{1}\right) \in H \times \mathbf{R}$ and $C^{\infty}$ diffeomorphisms $\varphi$ and $\psi$ such that the diagram

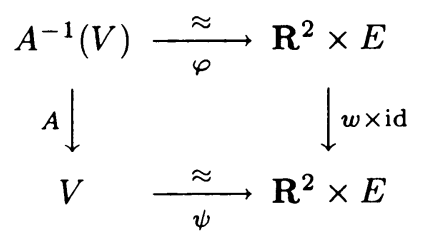


commutes, where $\varphi\left(0, \lambda_{1}\right)=(0,0,0)=\psi\left(0, \lambda_{1}\right), E$ is a closed subspace of $H$, and $w: \mathbf{R}^{2} \rightarrow \mathbf{R}^{2}$ is the map $w(t, \lambda)=\left(t^{3}-\lambda t, \lambda\right)$ defined by Hassler Whitney (cf. [BCT-2, (1.8)]) and $\varphi\left(0, \lambda_{1}\right)=(0,0,0)$. (Here $E$ is the orthogonal complement in $H$ of the first eigenspace of $\Delta$.) Figure 1 represents diagram (0.4) with the (one) vertical dimension corresponding to the infinite dimensional space $E$. This coordinate change has the disadvantage that it moves $\lambda$; on the other hand, $w \times$ id is a particularly simple and instructive form and we can use it to identify the numbers of solutions.

As a result of (0.4) the map $A: A^{-1}(V) \rightarrow V$ has the following structure: Its singular set $S A(2.5)$ is a real analytic submanifold of codimension one in $A^{-1}(V) \subset$ $H \times \mathbf{R}$, and $A$ maps $S A$ homeomorphically onto $A(S A)$, a topological submanifold of codimensional one in $V$. Thus $A(S A)$ separates $V$ into two components. If $(g, \lambda) \in V$ is in the component of $V-A(S A)$ that contains $(0, \mu)$ for any $\mu<\lambda_{1}$, then $A_{\lambda}(u)=g$ has exactly one solution (point inverse) $u$; if it is in the other component, then $A_{\lambda}(u)=g$ has precisely three solutions $u$.

Each point $(u, \lambda) \in A^{-1}(V)$ of $S A$ is either a fold point $((3.1),(3.2))$, i.e. $A$ at $(u, \lambda)$ is $C^{\infty}$ equivalent [BCT-2, (1.2)] to the map $F: \mathbf{R} \times \bar{E} \rightarrow \mathbf{R} \times \bar{E}$ defined by $F(t, v)=\left(t^{2}, v\right)$, or a cusp map $((3.1),(3.2))$, i.e. $A$ at $(u, \lambda)$ is $C^{\infty}$ equivalent to $G=w \times$ id. For each $(g, \lambda) \in A(S A), A_{\lambda}(u)=g$ has either two solutions or one, according as $(g, \lambda)$ is the image of a fold point or a cusp point. We can characterize the fold and cusp points of $A$ on $A^{-1}(V)$ as follows: For each $u \in S A_{\lambda}$, $\operatorname{dim} \operatorname{ker} D A_{\lambda}(u)=1$; let $0 \neq e \in \operatorname{ker} D A_{\lambda}(u)$. Then $(u, \lambda)$ is a fold [resp., cusp] point of $A$ if and only if $\int_{\Omega} u e^{3} \neq 0$ [resp., $=0$ ] (see (3.4), (3.5) and (3.6)).

Again for $A: A^{-1}(V) \rightarrow V$, the set of cusp points is a real analytic submanifold $\Gamma$ of codimension one in $S A$ (thus codimension two in $A^{-1}(V)$ ), $A(\Gamma)$ is a real analytic submanifold of codimension two in $V$, and the map $\Gamma \rightarrow A(\Gamma)$ by $A$ is a real analytic diffeomorphism. Also, the homeomorphism $S A \rightarrow A(S A)$ by $A$ is a real analytic diffeomorphism of $S A-\Gamma$ onto $A(S A-\Gamma)=A(S A)-A(\Gamma)$.

In the sequels $[\mathbf{C T}]$ and $[\mathbf{C D T}]$ (which uses $[\mathbf{C a C}]$ ) this investigation is extended. In particular, Hölder space analogs are discussed in [CDT].

The equation $\Delta u+\lambda u-u^{3}=0$ is known [B-2, $\left.\mathbf{A M}\right]$ to have precisely three solutions if $\lambda_{1}<\lambda<\lambda_{2}$. In this paper we consider $\Delta u+\lambda u-u^{3}=g$ for $g$ more general than 0 , and give the structure of the map.

Our paper is organized as follows. $\S 1$ gives the definition of the map abstract $A$ and examples, and $\S 2$ has necessary background results. $\S 3$ discusses the local fold and cusp structures for abstract $A$ and thus most of the examples, and it gives the main theorem. $\S 4$ shows how the general results can be applied to the von Kármán equations governing the combined bending and buckling of a thin elastic plate.

This paper, its predecessor [BCT-2], and its sequels [CT] and [CDT] involve both singularity theory and partial differential equations, and they are written to be accessible to those who are expert in only one of the fields. In particular, this paper has somewhat more detail than an expert in partial differential equations would require. Announcements of this work are given in [BCT-1] (addressed primarily to researchers in singularity theory) and [BCT-3] (addressed to experts in partial differential equations). The reader with limited background in partial differential equations may find it best to start with [BCT-1]. The singularity background for the present paper is developed in [BCT-2]; it gives abstract characterizations of 
the fold and cusp maps used in the present paper (and restated here as (3.1) and (3.2)).

Church and Timourian are grateful to E. N. Dancer and W. Allegretto for furnishing a number of references, and to E. N. Dancer for advice on what in an earlier draft was well known to experts in partial differential equations. Church thanks Syracuse University for research leave during 1986-87, and the University of Alberta for its hospitality and support during that period.

\section{Definition of the map abstract $A$ and examples.}

1.1. Notation. An ordered pair in $X \times Y$ is denoted by $(x, y)$, while the inner product of $x$ and $y$ in a Hilbert space $H$ (resp., $L^{2}(\Omega)$ ) is denoted by $\langle x, y\rangle_{H}$ (resp., $\left.\langle x, y\rangle_{2}\right)$. The norm of $x$ in $L^{p}(\Omega)$ is $\|x\|_{p}$. Real analytic [Z, (8.8), p. 362] is denoted by $C^{\omega}$. Assume throughout that $\Omega$ is a bounded connected open set in $\mathbf{R}^{n}$.

1.2. Definition. The abstract map $A$. Consider any Hilbert space $H$ over the real numbers and a map $A_{\lambda}: H \rightarrow H$ defined by

$$
A_{\lambda}(u)=u-\lambda L u+N(u)
$$

where $L$ and $N$ have the following properties:

(1) $L$ is a compact, selfadjoint, positive linear operator $\left(\langle L u, u\rangle_{H} \geq 0\right.$ and $=0$ only if $u=0$ ). It follows [De, pp. 349-350] that $H$ is separable and the eigenvalues $\lambda_{m}(m=1,2, \ldots)$ of $u=\lambda L u$ are positive, $\lambda_{m} \leq \lambda_{m+1}$, and (if $H$ is infinite dimensional) $\lambda_{m} \rightarrow \infty$ as $m \rightarrow \infty$. Let $\left\{u_{m}\right\}$ be an orthonormal basis of $H$ of eigenvectors.

(2) The first eigenvalue $\lambda_{1}$ is simple.

(3) (a) The map $N$ is $C^{k}(k=1,2, \ldots$ or $\infty$ or $\omega)$ such that $D N(u)$ is nonnegative selfadjoint $\left(\langle D N(u) \cdot v, v\rangle_{H} \geq 0\right.$ for every $\left.v \in H\right)$.

(b) If $\left\langle D N(u) \cdot u_{m}, u_{m}\right\rangle_{H}=0$ for some $m(m=1,2, \ldots)$, then $u=0$. [Statement $\left(\mathrm{b}_{1}\right)$ is $\left\langle D N(u) \cdot u_{1}, u_{1}\right\rangle_{H}=0$ implies $u=0$.]

(c) $k \geq 2$ and $D^{j} N(0)=0$ for $j=0,1,2$. [Statement $\left(c_{j}\right)$ for $j=0,1,2$ is $N$ is $C^{j}$ and $D^{j} N(0)=0$.]

(d) $k \geq 3$ and $\left\langle D^{3} N(u)(v, v, v), v\right\rangle_{H}>0$ for $0 \neq v \in H$.

(e) $D^{4} N(u) \equiv 0$. From Taylor's Theorem [Z, Theorem 4.A, p. 148] it follows that $N$ is real analytic, and assuming $(3)(\mathrm{c}),(3 !) N(u)=D^{3} N(0)(u, u, u)$, so that $2 D N(u) \cdot v=D^{3} N(0)(u, u, v)$.

We refer to a map $A_{\lambda}$ satisfying (1) and (3)(a) above, and to $A$ defined by $A(u, \lambda)=\left(A_{\lambda}(u), \lambda\right)$, as abstract $A_{\lambda}$ and $A$. Often a lemma will assume abstract $A$ and some of the above conditions, e.g. Lemma 2.7 requires abstract $A$ with (2), $(3)\left(b_{1}\right)$ and $(c)$ in addition.

In (1.3) we note that the map $A$ of $(0.1),(0.2)$ and (0.3) is an example of abstract $A$ satisfying all of the above properties, indeed (1.5) with $N$ compact if $n \leq 3$, and in (1.4) we give a class of examples more general than standard $A$.

1.3. ExAMPLE. The standard map $A$. Our main example of abstract $A(1.2)$ is the map $A$ of $(0.2)$ and equation (0.1); here $H$ is the Sobolev space $W_{0}^{1,2}(\Omega)$ ([BCT-1, §2] or [B-1, p. 28]), where $\Omega$ is a bounded open subset of $\mathbf{R}^{n}$ and $n \leq 4$. The operators $L$ and $N$ are defined by

$$
\langle L u, \varphi\rangle_{H}=\int_{\Omega} u \varphi \text { and }\langle N(u), \varphi\rangle_{H}=\int_{\Omega} u^{3} \varphi
$$


for all $\varphi \in C_{0}^{\infty}(\Omega)$, the space of $C^{\infty}$ real valued functions with compact support in $\Omega$. That $A_{\lambda}, L$, and $N$ are well defined follows from the Sobolev imbedding theorem [A, p. 97], the Hölder inequality [B-1, p. 28], the denseness of $C_{0}^{\infty}(\Omega)$ in $H$, and the Riesz representation theorem.

The Fréchet derivatives of $A_{\lambda}$ are defined by

$$
\begin{aligned}
& \left\langle D A_{\lambda}(u) \cdot \psi, \varphi\right\rangle_{H}=\int_{\Omega}\left[\nabla \psi \nabla \varphi-\lambda \psi \varphi+3 u^{2} \psi \varphi\right], \\
& \left\langle D^{2} A_{\lambda}(u)(\psi, \eta), \varphi\right\rangle_{H}=6 \int_{\Omega} u \psi \eta \varphi, \\
& \left\langle D^{3} A_{\lambda}(u)(\psi, \eta, \varsigma), \varphi\right\rangle_{H}=6 \int_{\Omega} \psi \eta \zeta \varphi
\end{aligned}
$$

for all $\varphi \in C_{0}^{\infty}(\Omega)$, and $D^{i} A_{\lambda} \equiv 0$ for $i \geq 4$. To show that the Fréchet derivatives exist, use the theorem relating Gateaux and Fréchet derivatives [B-1, (2.1.13), p. 68]. Since the Taylor series of $A_{\lambda}$ terminates, $A_{\lambda}$ is $C^{\omega}$ (real analytic) [Z, Definition (8.8), p. 362]. Condition (1.2)(3) results except for (3)(b).

By the Rellich imbedding theorem [A, p. 144], for $u \in H$ and sup over all $\varphi \in H$ with $\|\varphi\|_{H}=1$,

$$
\|L u\|_{H}=\sup _{\varphi}\langle L u, \varphi\rangle_{H}=\sup _{\varphi} \int_{\Omega} u \varphi \leq C\|u\|_{2}
$$

for some $C=C(\Omega)>0$, so $L$ is a compact linear operator and (1.2)(1) results. The eigenvalues of $u=\lambda L u$ are those of $\Delta u+\lambda u=0$ with null boundary conditions $(u \mid \partial \Omega=0)$; thus its first eigenvalue $\lambda_{1}$ is simple with eigenspace spanned by a $C^{\infty}$ function positive everywhere on $\Omega$ [GT, Theorem 8.38, Corollary 8.11, Theorem 8.21 , pp. $214,186,189]$. In fact the eigenfunctions $u_{m}$ of $-\Delta$ are real analytic [BJS, pp. 136, 207-210] so that the zeros of $u_{m}$ have measure 0 . Thus (1.2)(2) and (3)(b) result, so that the $A$ of $(0.3)$, which we call standard $A$, is an example of abstract $A$ satisfying $k=\omega$ and all the properties of (1.2), if $n \leq 4$.

1.4. EXAMPLE (a generalization of (1.3)). Consider $\Delta u+\lambda u-f(u)=g$ on a bounded domain $\Omega \subset \mathbf{R}^{n}(n \leq 3)$ with $\partial \Omega$ a $C^{\infty}$ manifold and $u \mid \partial \Omega=0$. Suppose that $f: \mathbf{R} \rightarrow \mathbf{R}$ is $C^{3}$ such that

(a) $f^{\prime}(s)>0$ for every $s \neq 0$;

(b) $f^{(j)}(0)=0$ for $j=0,1,2$; and

(c) $f^{(3)}(s)>0$.

Let $H=W_{0}^{1,2}(\Omega)$ and suppose that $f$ is so chosen that $N$ is $C^{3}$, e.g. $f^{(3)} \in L^{\infty}(R)$, where

$$
\left\langle D^{j} N(u)\left(v_{1}, v_{2}, \ldots, v_{j}\right), \varphi\right\rangle_{H}=\int_{\Omega} f^{(j)}(u) v_{1} v_{2} \cdots v_{j} \varphi \quad(j=0,1,2,3) .
$$

Define $A_{\lambda}: H \rightarrow H$ by

$$
\left\langle A_{\lambda}(u), \varphi\right\rangle_{H}=\int_{\Omega}[\nabla u \nabla \varphi-\lambda u \varphi+f(u) \varphi]
$$

then $A$ satisfies (1.2) with $k=3$, except for $(1.2)(3)(\mathrm{e})$. Of course equation $(0.1)$ satisfies the above properties. 
In [Sz] Szulkin considers a generalization of $\Delta u+\lambda u-f(u)=0$ on bounded $\Omega \subset \mathbf{R}^{n}$ with $u \mid \partial \Omega=0$. (He actually uses a space different from our $H$.) He assumes that $f(t)$ is convex for $t>0$, concave for $t<0, f(0)=f^{\prime}(0)=0$ and

$$
\lim _{t \rightarrow-\infty} f^{\prime}(t)=k_{-}>0, \quad \lim _{t \rightarrow+\infty} f^{\prime}(t)=k_{+}>0 \text {. }
$$

If we assume that $f$ is $C^{3}$, then $f$ satisfies (a), (b) and $\left(\mathrm{c}^{\prime}\right) f^{(3)}(0) \geq 0$.

1.5. LEMMA. If $f$ in (1.4) is $C^{4}, f^{(3)}$ and $f^{(4)}$ are bounded, and $n \leq 4$, the (nonlinear) operator $N$ is compact. In particular, for standard $A$ and $n \leq 3, N$ is compact.

We do not need hypotheses (1.4)(a) and (c).

ProOF. By Taylor's formula [Z, pp. 148-149] $f(s)=h(s) s^{3}$, where $h$ is $C^{1}$ and $h$ and $h^{\prime}$ are bounded by some $B>0$. For $u, v \in H$ with $\|u\|_{H},\|v\|_{H}<K$ and sup over $\varphi \in H$ with $\|\varphi\|_{H}=1$

$$
\begin{aligned}
\|N(u)-N(v)\|_{H} & =\sup _{\varphi}\langle N(u)-N(v), \varphi\rangle_{H} \\
& \leq \sup _{\varphi} \int_{\Omega} h(u)\left(u^{3}-v^{3}\right) \varphi+\sup _{\varphi} \int_{\Omega}(h(u)-h(v)) v^{3} \varphi \\
& \leq B \sup _{\varphi} \int_{\Omega}|u-v|\left|u^{2}+u v+v^{2}\right||\varphi|+B \sup _{\varphi} \int_{\Omega}|u-v|\left|v^{3}\right||\varphi| \\
& \leq C\|u-v\|_{5}
\end{aligned}
$$

by the Hölder inequality [B-1, p. 28] and the Rellich-Kondrachov imbedding theorem [A, p. 147], and the compactness of $N$ results.

1.6. REMARK. For the Szulkin example $(1.4), N$ is compact $(n=1,2, \ldots)$, as he notes [Sz, p. 103]. (From the boundedness of $f^{\prime}$, the Mean Value Theorem, and [GT, Lemma 7.6, p. 152],

$$
\left.\|N u-N v\|_{H}=\sup _{\varphi} \int_{\Omega} f^{\prime}(\xi)(u-v) \varphi \leq C\|u-v\|_{2} .\right)
$$

1.7. EXAMPLE (BERGER [B-2, pp. 695-697]). Let $\Omega$ be a bounded domain in $\mathbf{R}^{n}$ with boundary $\partial \Omega$, and consider the inhomogeneous nonlinear elliptic boundary value problem

$$
\begin{gathered}
L u-\lambda u+h(x) u^{2 p+1}=g(x), \\
D^{\alpha} u|\partial \Omega=0, \quad| \alpha \mid \leq m-1 .
\end{gathered}
$$

Here $h(x)$ is a positive smooth function on $\bar{\Omega}$, and $L$ is the formally selfadjoint linear elliptic operator of order $2 m$ defined by

$$
L u=(-1)^{m} \sum_{|\alpha|,|\beta| \leq m} D^{\alpha}\left\{a_{\alpha, \beta}(x) D^{\beta} u\right\},
$$

where the functions $a_{\alpha, \beta}(x)$ are smooth on $\bar{\Omega}(m=1,2, \ldots)$. Assume that the smallest eigenvalue $\mu_{1}$ of $L u=\mu u$ is simple, and $p>0$ is unrestricted if $2 m \geq n$ and strictly less than $2 m(n-2 m)^{-1}$ otherwise.

In [B-2] the author shows that the solutions of the above boundary value problem are in one-to-one correspondence with the solutions of an operator equation

$$
A_{\lambda+c_{2}}(u)=u-\left(\lambda+c_{2}\right) L u+N u=\tilde{g},
$$


where $A_{\lambda+c_{2}}: H \rightarrow H$ and $c_{2}$ is some real number. Here $H$ is the Sobolev space $W_{0}^{m, 2}(\Omega)$ with an inner product different from but equivalent to the usual one, and $L, N$, and $\tilde{g}$ are defined by

$$
\begin{gathered}
\langle L u, \varphi\rangle_{H}=\int_{\Omega} u \varphi, \quad\langle\tilde{g}, \varphi\rangle_{H}=\int_{\Omega} g \varphi \\
\langle N u, \varphi\rangle_{H}=\int_{\Omega} h(x) u^{2 p+1} \varphi
\end{gathered}
$$

for all $\varphi \in C_{0}^{\infty}(\Omega)$. The author proves that $L$ and $N$ satisfy the abstract assumptions $A$ and $B$ of [B-2, p. 690].

Now let $A_{\lambda+c_{2}}: H \rightarrow H$ be any operator given by (3) where $L$ and $N$ are abstract operators satisfying assumptions $A$ and $B$ of $[\mathrm{B}-2$, p. 690] and $p=1,2, \ldots$ The reader may verify that $A_{\lambda+c_{2}}$ is an example of abstract A; indeed it satisfies all of (1.2) except possibly for (1.2)(3)(b), (d), (e), and for $p=1$ it satisfies all but $(1.2)(3)(\mathrm{b})$.

Thus our specified example ((1) and (2)) is an example of abstract A, it satisfies all of (1.2) except for $(1.2)(3)(\mathrm{d}),(\mathrm{e})$, and for $p=1$ it satisfies all of (1.2).

1.8. EXAMPLE (BERGER [B-2, p. 697]). Let $\Omega$ be the unit disk in the plane, and consider the nonlinear elliptic boundary value problem

$$
\begin{gathered}
\Delta^{2} u-\lambda u+u^{2 p+1}=g \quad \text { on }|x|<1 \quad(p=1,2, \ldots), \\
u=\partial u / \partial n=0 \quad \text { on }|x|=1 .
\end{gathered}
$$

Since this problem is a special case of (1.7)(1) and (2), it also yields an example of abstract A (the last paragraph of (1.7)).

1.9. EXAMPLE. In $\S 4$ we study the von Kármán equations for the buckling of a thin planar elastic plate, compressed or stretched at each point of its edge. We use the form derived by the first author [B-3], and note in (4.6) that the resulting operator $A_{\lambda}$ satisfies many of the properties of abstract $A(1.2)$. In particular, in the case of compression only it satisfies all of (1.2) except possibly for (1.2)(2), (3)(a) and (b).

\section{Background results.}

2.1. Notation. Let $\lambda_{i}>0(i=1,2, \ldots)$ be the eigenvalues of $u=\lambda L u$ in (1.2)(1), given in increasing order and counting multiplicity, and let $u_{i}$ be corresponding eigenvectors. Let $E_{j}$ be the subspace of $H$ generated by $\left\{u_{1}, \ldots, u_{j}\right\}$, let $E_{0}=\{0\}$, and let $H_{j}$ be the orthogonal complement in $H$ of $E_{j}$. For $(x, v) \in E_{j}+H_{j}=H$, write

$$
A_{\lambda}(x, v)=\left(A_{\lambda, 1}(x, v), A_{\lambda, 2}(x, v)\right) \in E_{j}+H_{j} .
$$

2.2. LEMMA. For the abstract $C^{k}$ map $A$ of (1.2), $\lambda<\lambda_{j+1}$ and each fixed $x \in E_{j}$, the map $v \rightarrow A_{\lambda, 2}(x, v)$ of $H_{j}$ into $H_{j}$ is a $C^{k}$ diffeomorphism (onto) $(j=0,1, \ldots)$. 
ProOF. From [Di, (8.9), p. 167] and (1.2)(3a) for $\varphi \in H_{j}$ with $\|\varphi\|_{H}=1$ and $u=(x, v)$

$$
\begin{aligned}
\left\langle D A_{\lambda, 2}(x, v) \cdot \varphi, \varphi\right\rangle_{H} & =\left\langle D A_{\lambda}(u) \cdot \varphi, \varphi\right\rangle_{H} \\
& =\|\varphi\|_{H}^{2}-\lambda\langle L \varphi, \varphi\rangle_{H}+\langle D N(u) \cdot \varphi, \varphi\rangle_{H} \\
& \geq\|\varphi\|_{H}^{2}-\lambda\langle L \varphi, \varphi\rangle_{H} \geq\|\varphi\|_{H}^{2}\left(1-\lambda / \lambda_{j+1}\right) \\
& =1-\lambda / \lambda_{j+1}>0
\end{aligned}
$$

by the first two lines of the proof of [De, Proposition 27.1, pp. 349-350]. By the Lax-Milgram Lemma [B-1, p. 34] $D A_{\lambda, 2}(x, v)$ has a bounded inverse with norm at most $1 /\left[1-\lambda / \lambda_{j+1}\right]$, and by Hadamard's theorem [Sc, (1.22), p. 16] $v \rightarrow A_{\lambda, 2}(x, v)$ is a diffeomorphism of $H_{j}$ onto $H_{j}$.

\subsection{COROllary. For $\lambda<\lambda_{1}$, abstract $A$ is a $C^{k}$ diffeomorphism.}

The proof yields the same conclusion for the $A$ of [B-2] and that of Szulkin (1.4) $(n=1,2, \ldots)$.

2.4. REMARK. For abstract $A$, each $x \in E_{j}$, and $\lambda<\lambda_{j+1}(j=0,1, \ldots)$, let $\Lambda_{\lambda, 2}(x, \cdot): H_{j} \rightarrow H_{j}$ be the inverse of $A_{\lambda, 2}(x, \cdot)$ given in $(2.2)$, let $\Lambda_{\lambda}(x, w)=$ $\left(x, \Lambda_{\lambda, 2}(x, w)\right)$ where $w \in H_{j}$, and let $\Lambda(x, w, \lambda)=\left(x, \Lambda_{\lambda, 2}(x, w), \lambda\right)$. From (2.2) (as well as the Inverse Function Theorem [Di, (10.2.5), p. 268] and the Open Mapping Theorem [B-1, (1.3.23), p. 34]) the map

$$
B: E_{j} \times H_{j} \times\left(-\infty, \lambda_{j+1}\right) \rightarrow E_{j} \times H_{j} \times\left(-\infty, \lambda_{j+1}\right), \quad(x, v, \lambda) \rightarrow\left(x, A_{\lambda, 2}(x, v), \lambda\right)
$$

is a $C^{k}$ diffeomorphism; since $\Lambda=B^{-1}, \Lambda$ is a $C^{k}$ diffeomorphism. The map $A_{\lambda} \Lambda_{\lambda}$ has the form $A_{\lambda} \Lambda_{\lambda}(x, w)=\left(h_{j}(x, w, \lambda), w\right)$ and $A \Lambda(x, w, \lambda)=\left(h_{j}(x, w, \lambda), w, \lambda\right)$ for some $C^{k}$ map $h_{j}(x, w, \lambda)=h_{j}: E_{j} \rightarrow E_{j}$ and all $\lambda<\lambda_{j+1}$. For simplicity we shall write $h$ for $h_{1}$.

2.5. Definition. For a differentiable Fredholm map $\Phi: X \rightarrow Y$ of index 0 of one Banach space into another, its singular set $S \Phi$ is the set of all $x \in X$ such that $D \Phi(x)$ is not an isomorphism, i.e. does not have a bounded inverse defined on all of $Y$. For abstract $A$, from (2.4) and the finite dimensionality of each $E_{j}$,

$$
S A=\left\{(u, \lambda) \in H \times \mathbf{R}: \operatorname{ker} D A_{\lambda}(u) \neq\{0\}\right\}
$$

and $(u, \lambda) \in S A$ if and only if $u \in S A_{\lambda}$.

2.6. REMARK. For $u=0$ the only singular points of abstract $A$ assuming $(1.2)(3)\left(c_{1}\right)$ are at $\lambda=\lambda_{j}(j=1,2, \ldots)$.

Proof. If $(0, \lambda) \in S A$, then there exists nonzero $\psi \in \operatorname{ker} D A_{\lambda}(0)$. By Definition $(1.2)(3)\left(c_{1}\right)$

$$
0=\left\langle D A_{\lambda}(0) \cdot \psi, \varphi\right\rangle_{H}=\langle\psi-\lambda L \psi, \varphi\rangle_{H}
$$

for every $\varphi \in H$, so $\psi$ is an eigenvector of $L$ with eigenvalue $\lambda$.

Part (iii) of the following lemma is not used in this paper, but is needed for the sequel.

2.7. LEMMA. In (i) and (ii) consider abstract $A$ and assume $(1.2)(2),(3)\left(b_{1}\right)$, $\left(c_{1}\right)$.

(i) Then $S A_{\lambda_{1}}=\{0\}$ and $\operatorname{ker} D A_{\lambda_{1}}(0)$ is generated by $u_{1}(1.2)(2)$. 
(ii) If $A_{\lambda_{1}}$ is proper or surjective, then $A_{\lambda_{1}}$ and $A$ for $\lambda \leq \lambda_{1}$ are homeomorphisms. Thus, if $A_{\lambda_{1}}(u)=y$ has a solution $u$ for every $y \in H$, then it has a unique solution for every $y \in H$.

(iii) More generally, let $H$ be any Hilbert space, let $\lambda_{1} \in \mathbf{R}$, and let $A: H \times$ $\left(-\infty, \lambda_{1}\right] \rightarrow H \times\left(-\infty, \lambda_{1}\right]$ be a $C^{1}$ Fredholm map of index 0 such that $A(u, \lambda)=$ $\left(A_{\lambda}(u), \lambda\right), S A=\left\{\left(0, \lambda_{1}\right)\right\}, S A_{\lambda_{1}}=\{0\}$, and $A_{\lambda_{1}}(0)=0$. If $A\left[\right.$ resp., $\left.A_{\lambda_{1}}\right]$ is proper, then it is a homeomorphism (onto).

ProOF. (i) For $u, \varphi \in H$ with $\|\varphi\|_{H}=1$,

$$
\begin{aligned}
\left\langle D A_{\lambda_{1}}(u) \cdot \varphi, \varphi\right\rangle_{H} & =\|\varphi\|_{H}^{2}-\lambda_{1}\langle L \varphi, \varphi\rangle_{H}+\langle D N(u) \cdot \varphi, \varphi\rangle_{H} \\
& \geq\langle D N(u) \cdot \varphi, \varphi\rangle_{H} \geq 0
\end{aligned}
$$

and equality holds only if $(1.2)(2) \varphi$ is a multiple of $u_{1}$ and (1.2)(3)( $\left.\mathrm{b}_{1}\right) u=0$. Conclusion (i) results.

(ii) For $\lambda<\lambda_{2}$ there is a $C^{k}$ diffeomorphism $\Lambda$ such that (2.4)

$$
A \Lambda: \mathbf{R} \times E \times \mathbf{R} \rightarrow \mathbf{R} \times E \times \mathbf{R}, \quad(x, w, \lambda) \rightarrow(h(x, w, \lambda), w, \lambda) .
$$

For $\lambda \leq \lambda_{1}, S(A \Lambda)=\left\{\left(0,0, \lambda_{1}\right)\right\}$ and $S A_{\lambda_{1}}=\{0\}$ by (i) and (2.5) so that $D_{x} h(x, w, \lambda)$ has the same sign everywhere except that it is zero at $\left(0,0, \lambda_{1}\right)$. Thus for each fixed $w$ and $\lambda=\lambda_{1}, h$ is a homeomorphism of $\mathbf{R}$ onto an open interval of $\mathbf{R}$. If $A_{\lambda_{1}}$ is proper or surjective, then so is each such $h$, and thus each such $h$ is a homeomorphism of $\mathbf{R}$ onto $\mathbf{R}$. For $\lambda<\lambda_{1}, A \Lambda$ is a homeomorphism by (2.3). It follows that $A$ (for $\lambda \leq \lambda_{1}$ ) and $A_{\lambda_{1}}$ are injective, surjective, and continuous. Each is open except at the singular point, and openness there is readily proved. (We consider $A \Lambda$ for $\lambda \leq \lambda_{1}$ [resp., $\lambda=\lambda_{1}$ ], let $U$ be an open neighborhood of the singular point $\left(0,0, \lambda_{1}\right)$, and for $a<0<b$ let $L_{[a, b]}$ be the line segment $\left\{\left(x, 0, \lambda_{1}\right): a \leq x \leq b\right\}$. There exists $L_{[a, b]} \subset U$, each $h$ is a homeomorphism of $\mathbf{R}$ onto $\mathbf{R}$, and $h\left(0,0, \lambda_{1}\right)=0$; as a result there exist $c<0<d$ such that $0 \in L_{[c, d]} \subset h\left(L_{[a, b]}\right)$. Thus there is an open neighborhood $V$ of $\left(0, \lambda_{1}\right) \in E \times \mathbf{R}$ [resp., of $\left.\left(0, \lambda_{1}\right) \in E \times \lambda_{1}\right]$ such that $L_{[a, b]} \times V \subset U$ and (by the continuity of $A \Lambda$ )

$$
\left(0,0, \lambda_{1}\right) \in L_{[c, d]} \times V \subset A \Lambda\left(L_{[a, b]} \times V\right) \subset A \Lambda(U) .
$$

Thus $\left(0,0, \lambda_{1}\right) \in \operatorname{int} A \Lambda(U)$, so that $A \Lambda$ is open at $\left(0,0, \lambda_{1}\right)$.)

For (iii), let $B: X \rightarrow X$ be $A$ [resp., $A_{\lambda_{1}}$ ], and let $x=\left(0, \lambda_{1}\right)$ [resp., 0]. Since $B(x)=x$,

$$
B \mid\left(X-B^{-1}(x)\right): X-B^{-1}(x) \rightarrow X-\{x\}
$$

is a local diffeomorphism, and from the properness hypothesis it is a finite-to-one covering map [P, p. 128]. Since $B$ is a local diffeomorphism except at $x, B^{-1}(x)$ is discrete except possibly for $x$. Suppose that $\operatorname{dim} H \geq 2$ : then $B^{-1}(x)$ cannot separate $X\left(\left[\mathbf{H W}\right.\right.$, Theorem IV4, p. 48] and [K, p. 674]), so $X-B^{-1}(x)$ has only one component.

If $\operatorname{dim} H \geq 3$, then $X-\{x\}$ is simply connected $[\mathbf{K}$, p. 674; HW, Theorem VI6, p. 88]; thus the map (*) is a homeomorphism [M, Theorem 6.6, Exercise 6.1 , pp. 159, 160]. Suppose $B^{-1}(x)$ contains $x^{\prime} \neq x$. Choose disjoint connected open neighborhoods $U$ of $x^{\prime}$ and $V$ of $x$ with $U$ sufficiently small that $B$ maps $U$ homeomorphically onto an open neighborhood of $x$. Since $B(V)$ is connected and not $\{x\}$, the map $\left(^{*}\right)$ is not a homeomorphism, and a contradiction results. 
Thus $B^{-1}(x)=x$, and $B$ is continuous, injective, and surjective. Now let $y_{k} \rightarrow x$, $y_{k} \in X-x$, and note that $B^{-1}\left(y_{k}\right)$ is a single point $x_{k}$. Since $B$ is proper, $\bigcup_{k}\left\{x_{k}\right\} \cup\{x\}$ is compact, and it follows that $x_{k} \rightarrow x$. Thus $B^{-1}$ is continuous at $x$, and so $B$ is a homeomorphism.

Suppose $\operatorname{dim} H=2$ and $A_{\lambda_{1}}$ is proper. From the covering property $A_{\lambda_{1}}$ is topologically equivalent to the complex analytic function $g_{d}(z)=z^{d}(d=1,2, \ldots)$. If $S$ is the 2-disk of all $u \in H$ with $\|u\|_{H} \leq 1$, then $A_{\lambda_{1}}^{-1}(S)$ is a topological 2disk also containing 0 in its interior. For $\bar{\lambda}<\lambda_{1}$ let $C(\bar{\lambda})=A_{\lambda_{1}}^{-1}(S) \times\left[\bar{\lambda}, \lambda_{1}\right]$, let $\partial C(\bar{\lambda})=A_{\lambda_{1}}^{-1}$ (bdy $\left.S\right) \times\left[\bar{\lambda}, \lambda_{1}\right]$, and let $T$ be the set of $u \in H$ such that $\|u\|_{H} \leq 1 / 2$; there exists $\bar{\lambda}<\lambda_{1}$ such that $A(\partial C(\bar{\lambda})) \cap\left(T \times\left[\bar{\lambda}, \lambda_{1}\right]\right)=\varnothing$. Let $E$ be the component of $A^{-1}$ ((int $\left.\left.T\right) \times\left[\bar{\lambda}, \lambda_{1}\right]\right)$ containing $\left(0, \lambda_{1}\right)$; then (if $\bar{E}$ is the closure of $E$ in $H \times$ $\left.\left(-\infty, \lambda_{1}\right]\right) \bar{E} \subset C(\bar{\lambda})-\partial C(\bar{\lambda})$, and it follows that $f=A \mid E: E \rightarrow(\operatorname{int} T) \times\left[\bar{\lambda}, \lambda_{1}\right]$ is proper. Let $f_{\lambda}$ be the map of $E \cap(H \times\{\lambda\})$ into (int $\left.T\right) \times\{\lambda\}$ defined by $f$ (i.e. by $A$ ). Then each $f_{\lambda}$ is proper. For $\lambda<\lambda_{1}, f_{\lambda}$ is a proper local homeomorphism with simply connected range, so it is a homeomorphism; thus its degree is \pm 1 . But $f_{\lambda_{1}}$ is topologically equivalent to $g_{d}$, which has degree $\pm d$. It follows that $d=1$, and thus $A_{\lambda_{1}}$ is a homeomorphism. If we assume instead that $A$ is proper, the argument of the last three sentences (with $f_{\lambda}$ replaced by $A_{\lambda}$ ) shows that $A$ is a homeomorphism.

If $\operatorname{dim} H=1, D A_{\lambda}$ for $\lambda<\lambda_{1}$ has the same sign everywhere, so that $D A_{\lambda_{1}}$ has that sign except at its unique singular point 0 . Thus, if $A_{\lambda_{1}}$ is proper, it is a homeomorphism of $\mathbf{R}$ onto $\mathbf{R}$. If $A$ is proper, then each $A_{\lambda}$ for $\lambda \leq \lambda_{1}$ is a homeomorphism of $\mathbf{R}$ onto $\mathbf{R}$, so that $A$ is a homeomorphism.

2.8. Lemma. For $n \leq 3$ the standard (1.3) maps $A$ and each $A_{\lambda}$ are proper.

ProOf. It suffices to prove that standard $A$ is proper. Given $A\left(u_{n}, \lambda(n)\right) \rightarrow$ $(g, \lambda)$, it is enough to prove that $\left\{\left(u_{n}, \lambda(n)\right)\right\}$ has a convergent subsequence. Now $A\left(u_{n}, \lambda(n)\right)=\left(A_{\lambda(n)}\left(u_{n}\right), \lambda(n)\right)$, so $(1) \lambda(n) \rightarrow \lambda$ and $A_{\lambda(n)}\left(u_{n}\right)=g_{n} \rightarrow g$, and (2) it suffices to prove that $\left\{u_{n}\right\}$ has a convergent subsequence.

Since $\lambda_{1}>0\left((1.3)\right.$ and (1.2)(1)) and $A$ is a diffeomorphism for $\lambda<\lambda_{1}$ (2.3), we may suppose that $\lambda>0$ and each $\lambda(n)>0$. Now

$$
\begin{aligned}
\left\|g_{n}\right\|_{H}\left\|u_{n}\right\|_{H} & \geq\left\langle g_{n}, u_{n}\right\rangle_{H}=\left\langle A_{\lambda(n)}\left(u_{n}\right), u_{n}\right\rangle_{H} \\
& =\left\|u_{n}\right\|_{H}^{2}+\int_{\Omega}\left(u_{n}^{4}-\lambda(n) u_{n}^{2}\right) .
\end{aligned}
$$

Let $\Omega_{+}=\left\{x \in \Omega: \lambda(n)-\left(u_{n}(x)\right)^{2}>0\right\}$, and note that

$$
\int_{\Omega_{+}}\left(\lambda(n)-u_{n}^{2}\right) u_{n}^{2}+\left\|g_{n}\right\|_{H}\left\|u_{n}\right\|_{H} \geq\left\|u_{n}\right\|_{H}^{2} .
$$

Now $\lambda(n) m\left(\Omega_{+}\right) \geq\left\|u_{n}\right\|_{2, \Omega_{+}}^{2}$, where $m\left(\Omega_{+}\right)$is the Lebesgue measure of $\Omega_{+}$,

$$
\int_{\Omega_{+}}\left(\lambda(n)-u_{n}^{2}\right) u_{n}^{2} \leq \int_{\Omega_{+}} \lambda(n) u_{n}^{2}=\lambda(n)\left\|u_{n}\right\|_{2, \Omega_{+}}^{2},
$$

and it follows from (3) that

$$
\lambda(n)^{2} m(\Omega)+\left\|g_{n}\right\|_{H}\left\|u_{n}\right\|_{H} \geq\left\|u_{n}\right\|_{H}^{2} .
$$


From (1), $\left\|g_{n}\right\|_{H}$ and $|\lambda(n)|$ have bounds $K_{1}$ and $K_{2}$, respectively, so

$$
K_{2}^{2}\left\|u_{n}\right\|_{H}^{-1} m(\Omega)+K_{1} \geq\left\|u_{n}\right\|_{H} .
$$

Thus $1+K_{2}^{2} m(\Omega)+K_{1} \geq\left\|u_{n}\right\|_{H}$ for all sufficiently large $n$; hence (4) the $u_{n}$ are bounded in $H$.

Now

$$
g_{n}=A_{\lambda(n)}\left(u_{n}\right)=u_{n}-\lambda(n) L\left(u_{n}\right)+N\left(u_{n}\right),
$$

where $L: H \rightarrow H$ and $N: H \rightarrow H$ are the compact maps of (1.3) and (1.5). (It is here that the assumption $n \leq 3$ is required.) From (4) there is a subsequence $u_{n(m)}$ such that $L\left(u_{n(m)}\right)$ and $N\left(u_{n(m)}\right)$ are convergent (in $H$ ) and from (1) $g_{n(m)}$ converges; thus from (5) $u_{n(m)}$ converges in $H$ so that (2) is true as desired.

2.9. REMARK. Suppose that $A$ is as given in (1.4) with

(i) $f^{(3)}(s) \geq f^{(3)}(0)>0$ for all $s \in \mathbf{R}$ and

(ii) $f$ is such that $N$ is compact (e.g. $f$ is $C^{1}$ and $f^{\prime}$ is bounded (1.6), or $n \leq 3$, $f$ is $C^{4}$, and $f^{(3)}$ and $f^{(4)}$ are bounded (1.5)).

Then $A$ and $A_{\lambda}$ are proper.

PROOF. There exists a continuous function $h: \mathbf{R} \rightarrow \mathbf{R}$ such that $f(s)=h(s) s^{3}$ and $h(0)=f^{(3)}(0) / 6 \neq 0$ by Taylor's formula $[\mathrm{Z}$, Theorem 4.A, p. 148]. In the proof of (2.8) make the following changes: In formula (3) replace $\int_{\Omega} u_{n}^{4}$ by $\int_{\Omega} h\left(u_{n}\right) u_{n}^{4}$ and define

$$
\Omega_{+}=\left\{x \in \Omega: \lambda(n)-h\left(u_{n}\right)\left(u_{n}(x)\right)^{2}>0\right\} .
$$

From (i) it follows that $\lambda(n) m\left(\Omega_{+}\right) \geq h(0)\left\|u_{n}\right\|_{2, \Omega_{+}}^{2}$.

2.10. Corollary. For $\lambda \leq \lambda_{1}$ and $n \leq 3$ standard $A$ and each $A_{\lambda}$ are homeomorphisms.

Use (2.8) and (2.7)(ii). Thus for $\lambda \leq \lambda_{1}$ and any $\tilde{g} \in H, A_{\lambda}(u)=\tilde{g}$ has a unique solution $u \in H$. In other words the equation

$$
\Delta u+\lambda u-u^{3}=g
$$

has unique (weak) solutions for $\lambda \leq \lambda_{1}$.

3. Local structure and the main theorem.

3.1. Definitions [BCT-2, (1.4) and (1.6)]. Let $A: E_{1} \rightarrow E_{2}$ be a $C^{k}(k=2,3, \ldots$ or $\infty$ or $\omega)$ map germ [BCT-2, (1.1)] at $\bar{u}$ in a Banach space $E_{1}$ such that

(0) $A$ is Fredholm [BCT-2, (1.3)] with index 0, i.e. $D A(\bar{u})$ is a Fredholm linear operator, and

(1) dim ker $D A(\bar{u})=1$ (and therefore range $D A(\bar{u})$ has codimension one).

DEFinition. Then $A$ is a fold if and only if

(2) for some (and hence for any) nonzero element $e \in \operatorname{ker} D A(\bar{u})$

$$
D^{2} A(\bar{u})(e, e) \notin \text { range } D A(\bar{u}) .
$$

Definition. The germ $A$ is a cusp [resp., intrinsic cusp] if and only if $k \geq 3$,

(2) for some (and hence for any) nonzero element $e \in \operatorname{ker} D A(\bar{u})$,

$$
D^{2} A(\bar{u})(e, e) \in \text { range } D A(\bar{u}),
$$


(3) for some $\omega \in E_{1}$,

$$
D^{2} A(\bar{u})(e, \omega) \notin \text { range } D A(\bar{u}),
$$

and

(4) $D^{2}(A \mid S A)(\bar{u})(e, e) \notin$ range $D(A \mid S A)(\bar{u})$ [resp., $(\tilde{4})$

$$
\left.D^{3} A(\bar{u})(e, e, e)-3 D^{2} A(\bar{u})\left(e,(D A(\bar{u}))^{-1}\left(D^{2} A(\bar{u})(e, e)\right)\right) \notin \text { range } D A(\bar{u})\right] .
$$

DEFINITION. If the germ $A$ satisfies (3) with $\omega \neq e$ [resp., (2) and (3)] for a cusp (with $k \geq 2$ ), then it is called good [resp., a precusp].

These concepts are well defined and invariant under coordinate change [BCT-2, (3.2), (3.5) and (3.6)], and cusp and intrinsic cusp are equivalent [BCT-2, (3.9)]. For the invariance of good use the proof of [BCT-2, (3.2)]. For "precusp" replaced by "good" [BCT-2, (3.3)] is true with (ii) and (b) deleted.

DEFINITION. Given a map $A$, a point $\bar{u}$ in its domain is called a fold point [resp., cusp point, precusp point, good point], if the germ of $A$ at $\bar{u}$ is a fold [resp., cusp, precusp, good].

3.2. ThEOREM [BC, p. 950; BCT-2, (1.5), (1.7)]. Let $A: E_{1} \rightarrow E_{2}$ be a $C^{k}(k=2,3, \ldots$ or $\infty)$ map germ at $\bar{u}$ in a Banach space $E_{1}$.

If $A$ is a fold, then $A$ is $C^{k-2}$ equivalent [BCT-2, (1.2)] to $F: \mathbf{R} \times E \rightarrow \mathbf{R} \times E$, $(t, v) \rightarrow\left(t^{2}, v\right)$ at $(0,0)$.

If $k \geq 3$ [resp., $k=\infty$ ] and $A$ is a cusp or intrinsic cusp, then $A$ is $C^{0}$ [resp., $\left.C^{\infty}\right]$ equivalent to $G: \mathbf{R}^{2} \times E \rightarrow \mathbf{R}^{2} \times E,(t, \lambda, v) \rightarrow\left(t^{3}-\lambda t, \lambda, v\right)$ at $(0,0,0)$.

The map $G=w \times$ id, where $w: \mathbf{R}^{2} \rightarrow \mathbf{R}^{2}$ was [BCT-2, (1.8)] defined by H. Whitney and is the first on Thom's list of elementary catastrophes. For pictures of $w$ see [GG, p. 147].

3.3. REMARKS. By (2.2) (and (2.4)) each abstract $A_{\lambda}$ (1.2), and thus abstract $A$ itself, is a Fredholm map of index 0 [BCT-2, (1.3)]. Let $\bar{u} \in S A_{\lambda}$ (2.5) and let $\lambda<\lambda_{2}$. Assuming (1.2)(2), the eigenspace of $\lambda_{1}$ is one dimensional, and it follows from (2.2) that dim $\operatorname{ker} D A_{\lambda}(\bar{u})=1$. Whenever $\operatorname{dim} \operatorname{ker} D A_{\lambda}(\bar{u})=1$, we may define $e=e(\bar{u}, \lambda) \in \operatorname{ker} D A_{\lambda}(\bar{u})$ with $\|e\|_{H}=1$.

The following lemmas (3.4), (3.5) and (3.6) do not need all of the hypotheses of abstract $A$, viz. $D N(\bar{u})$ in $(1.2)(3)(\mathrm{a})$ is not required to be nonnegative.

3.4. Proposition. For $\lambda<\lambda_{2}$ a singular point $\bar{u}$ of abstract $A_{\lambda}$ [resp., $(\bar{u}, \lambda)$ of abstract $A]$ is a fold point (3.1) if and only if there exists a nonzero $e \in$ ker $D A_{\lambda}(\bar{u})$ such that $\left\langle D^{2} N(\bar{u})(e, e), e\right\rangle_{H} \neq 0$ (i.e. for standard $A$ (1.3) if and only if $\left.\int_{\Omega} \bar{u} e^{3} \neq 0\right)$.

More generally, for abstract $A$ and $A_{\lambda}$ for any $\lambda$ with $\operatorname{dim} \operatorname{ker} D A_{\lambda}(\bar{u})=1$, the same conclusion results.

ProOF. For all $\varphi \in H, 0=\left\langle D A_{\lambda}(\bar{u}) \cdot e, \varphi\right\rangle_{H}=\left\langle D A_{\lambda}(\bar{u}) \cdot \varphi, e\right\rangle_{H}$ by $(1.2)(1)$ and (3)(a); thus range $D A_{\lambda}(\bar{u})$ is the orthogonal complement of $e$. Condition (2) of a fold point $\bar{u}(3.1)$ is $\left\langle D^{2} A_{\lambda}(\bar{u})(e, e), e\right\rangle_{H} \neq 0$, which yields the conclusion for $A_{\lambda}$.

For $(u, \lambda) \in H \times \mathbf{R}$, write $A(u, \lambda)$ as $\left(A_{1}(u, \lambda), A_{2}(u, \lambda)\right) \in H \times \mathbf{R}$, so that $A_{1}(u, \lambda)=A_{\lambda}(u)$ and $A_{2}(u, \lambda)=\lambda$. For $\left(v_{1}, v_{2}\right) \in H \times \mathbf{R}$

$$
\begin{aligned}
D A(u, \lambda) \cdot\left(v_{1}, v_{2}\right) & =\left(D_{u} A_{1}(u, \lambda) \cdot v_{1}+D_{\lambda} A_{1}(u, \lambda) \cdot v_{2}, v_{2}\right) \\
& =\left(D A_{\lambda}(u) \cdot v_{1}-v_{2} L u, v_{2}\right)
\end{aligned}
$$


by [Di, $(8.91)$, p. 167]. Thus $\operatorname{ker} D A(\bar{u}, \lambda)$ is spanned by $(e, 0)$, and the orthogonal complement of range $D A(\bar{u}, \lambda)$ has dimension one (3.3) and is generated by $(e, a) \in$ $H \times \mathbf{R}$, where $a=\langle e, L \bar{u}\rangle_{H}$. (For standard $A, a=\int_{\Omega} \bar{u} e$.) Since

$$
D^{2} A(u, \lambda)\left(\left(v_{1}, v_{2}\right),\left(w_{1}, w_{2}\right)\right)=(x, 0)
$$

where $x \in H$ is defined by

$$
x=D^{2} N(u)\left(v_{1}, w_{1}\right)-w_{2} L v_{1}-v_{2} L w_{1},
$$

condition (2) of a fold (3.1)

$$
0 \neq\left\langle D^{2} A(\bar{u}, \lambda)((e, 0),(e, 0)),(e, a)\right\rangle_{H \times \mathbf{R}}
$$

reduces to that for $A_{\lambda}$. This yields the conclusion for $A$.

3.5. LEMMA. For $\lambda<\lambda_{2}$ a singular point $(\bar{u}, \lambda)$ of abstract $A$ assuming $(1.2)(2)$ is good (3.1). Thus it is a precusp point if and only if it is not a fold point (for standard $A$ if and only if $\int_{\Omega} u e^{3}=0$ ).

More generally, this holds for any $(\bar{u}, \lambda)$ with $\operatorname{dim} \operatorname{ker} D A_{\lambda}(\bar{u})=1$.

Proof. Condition (3) of a good point $\bar{u}$ (3.1) is that for some $\omega \in H \times \mathbf{R}$

$$
\left\langle D^{2} A(\bar{u}, \lambda)((e, 0), \omega),(e, a)\right\rangle_{H \times \mathbf{R}} \neq 0 .
$$

(See (3.3) and (3.4).) Let $\omega=(0,1) \in H \times \mathbf{R}$; then (3) becomes $-\langle L e, e\rangle_{H} \neq 0$, which holds by (1.2)(1) since $e \neq 0$ (3.3). The conclusion results from (3.4) (and its proof).

3.6. Proposition. Consider abstract $A$, assuming $(1.2)(2),(3)\left(c_{2}\right)$ and (d). The point $\left(0, \lambda_{1}\right) \in H \times \mathbf{R}$ is a cusp point of $A$. Any singular point $(\bar{u}, \lambda)$ sufficiently near $\left(0, \lambda_{1}\right)$ is a cusp point if and only if $\left\langle D^{2} N(\bar{u})(e, e), e\right\rangle_{H}=0$ (for standard $A$, if and only if $\int_{\Omega} \bar{u} e^{3}=0$ ).

More generally, if $\lambda_{j}$ is a simple eigenvalue (so that $\operatorname{dim} \operatorname{ker} D A_{\lambda_{j}}(0)=1$ ), then the same conclusion holds for $\left(0, \lambda_{j}\right)(j=1,2, \ldots)$.

Thus in a sufficiently small neighborhood of $\left(0, \lambda_{1}\right)$ (or such $\left(0, \lambda_{j}\right)$ ) in $H \times \mathbf{R}$ all the singular points of abstract $A$ are either folds or cusps; for standard $A$ this is specified by $\int_{\Omega} \bar{u} e^{3} \neq 0$ or $=0$.

PrOof. Condition ( $\tilde{4})$ for (intrinsic) cusp point (3.1) $(\bar{u}, \lambda)$ of abstract $A$ states that

$$
\begin{aligned}
& \left\langle D^{3} A(\bar{u}, \lambda)((e, 0),(e, 0),(e, 0)),(e, a)\right\rangle_{H \times \mathbf{R}} \\
& \quad-3\left\langle D^{2} A(\bar{u}, \lambda)((e, 0),(y, 0)),(e, a)\right\rangle_{H \times \mathbf{R}} \neq 0,
\end{aligned}
$$

where $(y, 0) \in(D A(\bar{u}, \lambda))^{-1}\left(D^{2} A(\bar{u}, \lambda)((e, 0),(e, 0))\right)$; for abstract $A$ this is the same condition with $A$ replaced by $N$ except that $(D A(\bar{u}, \lambda))^{-1}$ remains and for standard $A$ it is $\int_{\Omega} e^{4}-3 \int_{\Omega} \bar{u} e^{2} y \neq 0$. By (1.2)(3)( $\left.\mathrm{c}_{2}\right)$ and (d) condition ( $\left.\tilde{4}\right)$ for abstract $A$ is satisfied at $(u, \lambda)=\left(0, \lambda_{1}\right)$, and by $(1.2)(2),(3)\left(c_{2}\right),(3.4)$ and (3.5) $\left(0, \lambda_{1}\right)$ is a precusp point, so it is a cusp point $(3.1)$.

Since abstract $A$ is $C^{k}(k \geq 3)$ by $(1.2)(3)(\mathrm{d})$, in some neighborhood $U$ of $\left(0, \lambda_{1}\right)$ the number of condition $(\tilde{4})$ above is also nonzero, so that in $U$ every precusp point is a cusp point. By (3.4) and (3.5) a singular point $(\bar{u}, \lambda) \in U$ of $A$ is a. cusp point if and only if $\left\langle D^{2} N(\bar{u})(e, e), e\right\rangle_{H}=0$. 
3.7. Lemma. Suppose that $X$ and $Y$ are $C^{k}$ Banach manifolds, and $A: X \rightarrow Y$ is a $C^{k}(k \geq 3$ [resp., $\left.k=\infty]\right)$ proper map which is Fredholm of index 0 . Suppose that $y \in Y$ and $A^{-1}(y) \cap S A=\{x\}$, where $x$ is a cusp point (3.1) of $A$. Then there is a connected open neighborhood $V$ of $y$ such that $A^{-1}(V)$ consists of components $U_{i}$ $(i=0,1, \ldots, m ; m=0,1, \ldots)$ such that $x \in U_{0}, A: U_{i} \rightarrow V$ is a $C^{k}$ diffeomorphism (onto) if $i \neq 0$, and is $C^{0}\left[\right.$ resp., $\left.C^{\infty}\right]$ equivalent at $x$ to $w \times \mathrm{id}(3.2)$ at $(0,0,0)$ if $i=0$.

If $A^{-1}(y) \cap S A=\varnothing$, the conclusion results with $U_{0}$ omitted.

PROOF. Since $A^{-1}(y)$ is discrete (from (3.2)) and $A$ is proper, $A^{-1}(y)=$ $\left\{x_{0}, x_{1}, \ldots, x_{m}\right\}$, where $x=x_{0}$ and $m=0,1, \ldots$ There are disjoint open neighborhoods $W_{i}$ of $x_{i}$ such that (1) $A: W_{i} \rightarrow A\left(W_{i}\right)$ is a $C^{k}$ diffeomorphism if $i \neq 0$, and is $C^{0}$ [resp., $C^{\infty}$ ] equivalent to $w \times$ id if $i=0(3.2)$. Thus each $A\left(W_{i}\right)$ is open in $Y$. Choose $V_{r}$ open neighborhoods of $y$ in $Y$ such that $\bar{V}_{1} \subset \bigcap_{i} A\left(W_{i}\right), \bar{V}_{r+1} \subset V_{r}$ $(r=1,2, \ldots)$, and $\operatorname{diam}\left(V_{r}\right) \rightarrow 0$. Let $U_{r, i}=A^{-1}\left(V_{r}\right) \cap W_{i}$, so that $\bar{U}_{1, i} \subset W_{i}$. We may choose the $V_{r}$ so that (2) $A \mid U_{r, 0}: U_{r, 0} \rightarrow V_{r}$ is $C^{0}$ [resp., $C^{\infty}$ ] equivalent to $w \times$ id (and, of course, $A \mid U_{r, i}: U_{r, i} \rightarrow V_{r}$ is a $C^{k}$ diffeomorphism).

Suppose that $A^{-1}\left(V_{r}\right) \neq \bigcup_{i} U_{r, i}$ for all $r(r=1,2, \ldots)$; then there exist $u_{r} \in$ $A^{-1}\left(V_{r}\right)-\bigcup_{i} U_{r, i}$. From (1) and (2) $u_{r} \notin \bigcup_{i} W_{i}$, while $A\left(u_{r}\right) \in V_{r}$, so that (3) $A\left(u_{r}\right) \rightarrow y$. Since $A$ is proper and $\left\{A\left(u_{r}\right): r=1,2, \ldots\right\} \cup\{y\}$ is compact, there is a subsequence $\left\{u_{r(j)}\right\}$ in $X$ such that $u_{r(j)} \rightarrow u$ for some $u \in X$. Because each $u_{r(j)} \in X-\bigcup_{i} W_{i}, u \in X-\bigcup_{i} W_{i}$. Now $A(u)=y, A^{-1}(y)=\left\{x_{0}, \ldots, x_{m}\right\} \subset \bigcup_{i} W_{i}$, and a contradiction results. Thus, for $r$ sufficiently large, $A^{-1}\left(V_{r}\right)=\bigcup_{i} U_{i, r}$, and the desired conclusion results from (2).

3.8. THEOREM. Suppose that abstract A, assuming $(1.2)(2),(3)\left(\mathrm{b}_{1}\right)(\mathrm{c})$ and (d), is a proper map and is $C^{3}\left[\right.$ resp., $\left.C^{\infty}\right]$. Then there exists a connected open neighborhood $V$ of $\left(0, \lambda_{1}\right) \in H \times \mathbf{R}$ and homeomorphisms $\left[\right.$ resp., $C^{\infty}$ diffeomorphisms] $\varphi$ and $\psi$ such that the diagram

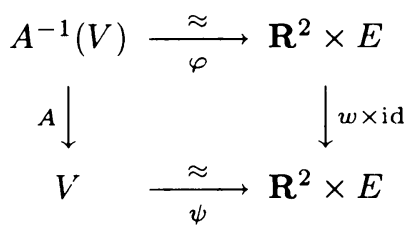

commutes, where $\varphi\left(0, \lambda_{1}\right)=(0,0,0)=\psi\left(0, \lambda_{1}\right), E$ is a closed subspace of $H$, and $w(t, \lambda)=\left(t^{3}-\lambda t, \lambda\right)(c f .(3.2))$.

ProOF. Since $\left(0, \lambda_{1}\right) \in H \times \mathbf{R}$ is a cusp point of $A$ by (3.6), and $A_{\lambda_{1}}$ is a homeomorphism by (2.7)(ii), the conclusion results from (3.7).

3.9. Corollary. Standard $A$ with $n \leq 3$ satisfies the $C^{\infty}$ conclusion of (3.8).

ProOF. Use (1.3), (2.8) and (3.8).

Thus, for each $g$ near 0 and $\lambda$ near $\lambda_{1}, A_{\lambda}(u)=g$ has either one, two, or three solutions and the bifurcation is thus described by (3.9). See the introduction for a more complete discussion.

3.10. REMARK (Corollary of (3.4), (3.6), and (3.8)). For $\Delta u+\lambda u-f(u)=g$ with $f$ satisfying (1.4), a singular point $(\bar{u}, \lambda)$ with $\lambda<\lambda_{2}$ of the resulting map $A$ 
of (1.4) is a fold point of $A$ (and thus of $A_{\lambda}$ ) if and only if $\int_{\Omega} f^{\prime \prime}(\bar{u}) e^{3} \neq 0$, and, for $(u, \lambda)$ sufficiently near $\left(0, \lambda_{1}\right)$, is a cusp point of $A$ if and only if this integral is zero. If $A$ is proper, then it satisfies the hypotheses and thus the conclusion of (3.8)

3.11. REMARK For standard $A_{\lambda}$ with $\lambda<\lambda_{2}$ and for $0 \neq \bar{u} \in H$, the ray $\{c \bar{u}: c \geq 0\}$ meets the singular set $S A_{\lambda}$ in at most one point.

PROOF. Note that $c \bar{u} \in S A_{\lambda}$ if and only if $D A_{\lambda}(\bar{u}) \cdot v=0$ for some $0 \neq v \in H$, i.e. if and only if $\lambda$ is an eigenvalue of $-\Delta v+\left(3(c \bar{u})^{2}-\lambda\right) v=0$ with $v \mid \partial \Omega=0$. Since the $m$ th eigenvalue $\lambda_{m}(c \bar{u})$ of that equation is an increasing function of $c \geq 0$, $\lambda_{m}(c \bar{u}) \geq \lambda_{m}(0)=\lambda_{m}$ (the $m$ th eigenvalue of $\Delta v+\lambda v=0$ with $v \mid \partial \Omega=0$ ) and $\lambda_{2}>\lambda$, the only possible eigenvalue $\lambda=\lambda_{1}(c \bar{u})$ and it is achieved for at the most one value of $c$.

In the sequel [CT] further information in this direction is given.

3.12. REMARK. Some other papers in which cusp singularities are discussed in connection with differential equations are $[\mathbf{C a D}-1, \mathbf{C a D - 2}, \mathbf{L M}, \mathbf{M c}, \mathbf{M c S}, \mathbf{R - 1}$ and R-2]. In particular, if $H=\left\{u \in W^{1,2}[0,1]: u(0)=u(1)\right\}$ and $\Phi: H \rightarrow L^{2}(0,1)$ is defined by $\Phi(u)=D u+u-u^{3}$, then (using the results of $[\mathrm{CaD}-1]$ and $[\mathrm{CaD}$ 2]) Cafagna and Church $[\mathbf{C a C}]$ have proved that $\Phi$ is a global cusp, i.e. there are homeomorphisms $\alpha$ and $\beta$ such that $\Phi=\beta^{-1}(w \times$ id $) \alpha$.

4. The von Kármán equations. Here we consider the bending and buckling of a thin planar elastic plate of arbitrary shape $\Omega \subset \mathbf{R}^{2}$, acted on by compressive and stretching forces on its boundary. These deformations are described by the von Kármán equations; for background information see [B-1, pp. 11-12, 97-99, 177-182; B-4, B-3, CR, St; An, especially pp. 271-273] and their references. In this section we show how the concepts of folds and cusps describe the deformations of the plate.

4.1. Definition. Let $H=W_{0}^{2,2}(\Omega)$, where $\Omega \subset \mathbf{R}^{2}$ is a bounded domain with boundary $\partial \Omega$ a $C^{5}$ manifold [BF-2, p. 229], and define $A_{\lambda}: H \rightarrow H$ by $A_{\lambda}(u)=u-\lambda L u+C u$, where $u-\lambda L u+C u=0$ constitutes the von Kármán equations for the plate (as a single equation) in the form given by Berger [B-4]. If we allow a force $p$ perpendicular to the plane of the plate and define $g \in H$ by $\langle g, \varphi\rangle_{H}=\int_{\Omega} p \varphi$ for all $\varphi \in H$, then the von Kármán equations become $A_{\lambda}(u)=g$ [B-3, p. 142 bottom and p. 423, Lemma A]. Define $A: H \times \mathbf{R} \rightarrow H \times \mathbf{R}$ by $A(u, \lambda)=\left(A_{\lambda}(u), \lambda\right)$. Throughout $\S 4, A$ and $A_{\lambda}$ refer to these functions.

4.2. LEMMA [B-4; B-3, Lemma A, p. 143]. (i) There is a bounded symmetric bilinear function $C$ of $H \times H$ into $H$ such that $C u=C(u, C(u, u))$ and, for some $F_{0} \in H, L u=C\left(F_{0}, u\right)$;

(ii) $\langle C u, u\rangle_{H}=\|C(u, u)\|_{H}^{2} \geq 0$ and equals 0 if and only if $u=0$;

(iii) $\langle C(u, v), w\rangle_{H}$ is symmetric in $u, v$ and $w$;

(iv) $L$ is a compact selfadjoint linear operator. 
4.3. LEMMA. The function $A_{\lambda}$ is real analytic [B-3, Theorem 2, p. 145] with:

(i) $D A_{\lambda}(u) \cdot v=v-\lambda L v+D C(u) \cdot v$, where

$$
D C(u) \cdot v=C(v, C(u, u))+C(u, C(v, u))+C(u, C(u, v))
$$

(ii)

$$
\begin{aligned}
D^{2} A_{\lambda}(u)(v, w) & =D^{2} C(u)(v, w) \\
& =2 C(u, C(v, w))+2 C(v, C(w, u))+2 C(w, C(u, v))
\end{aligned}
$$

(iii)

$$
\begin{aligned}
D^{3} A_{\lambda}(u)(v, w, x) & =D^{3} C(u)(v, w, x) \\
& =2 C(v, C(w, x))+2 C(w, C(x, v))+2 C(x, C(v, w)) ;
\end{aligned}
$$

and

(iv) $D^{j} A_{\lambda}(u)=D^{j} C(u) \equiv 0$ for $j \geq 4$.

(v) Moreover, $D C(u)$ and $D A_{\lambda}(u)$ are selfadjoint, and [B-3, Theorem 2, p. 145] $A_{\lambda}: H \rightarrow H$ is a Fredholm map of index 0 .

For (v) use (i) with (4.2)(iii). Here real analytic is defined as in [Z, p. 362], and the analyticity of $A_{\lambda}$ follows from (iv).

4.4. Lemma [B-3, Theorem 1, p. 144]. The map $A: H \times \mathbf{R} \rightarrow H \times \mathbf{R}$ is proper.

PROOF. That each $A_{\lambda}$ is proper is proved [B-3, Theorem 1, p. 144], and we generalize that proof. Specifically, given $\left(g_{n}, \lambda(n)\right) \rightarrow(g, \lambda)$ and $u_{n}$ with $A_{\lambda(n)}\left(u_{n}\right)=g_{n}$, we wish to find a subsequence $u_{n(k)}$ and $u$ with $u_{n(k)} \rightarrow u$. In the proof of [B-3, Theorern 1, p. 144], replace $\lambda$ by $\lambda_{n}$ in displays (4) and (5). In display (6) replace $|\lambda|$ by $|\lambda|+1$, consider $n$ sufficiently large that $\left|\lambda_{n}\right| \leq|\lambda|+1$, and set $\varepsilon=|\lambda|+1$.

4.5. REMARK $[\mathbf{B}-4, \S 1.4$, p. $690 ;$ p. 701 , first paragraph]. If one compresses the plate everywhere on its edge $\partial \Omega$, and nowhere stretches it, then all the eigenvalues of $L$ are positive, so $L$ is a positive operator $(\langle L e, e\rangle>0$ for $0 \neq e \in H)$.

4.6. LEMMA. The map $A_{\lambda}$ of (4.1) satisfies all the properties of abstract $A_{\lambda}$ (1.2) where $C u=N u$, with the following possible exceptions: (i) (only positivity of $L$ fails), (2), (3)(a) (only nonnegativeness of $D C(u)$ fails), (3)(b). In case of compression only (4.5), condition (1) is completely satisfied.

ProOF. Conclusion (1) (without positivity) is (4.2)(iv); (3)(a) (without nonnegativity) is $(4.3)(\mathrm{v}) ;(3)(\mathrm{c})$ follows from $(4.2)(\mathrm{i})$ and $(4.3)(\mathrm{i})$ and (ii); (3)(d) follows from (4.3)(iii) and (4.2)(ii); (3)(e) is (4.3)(iv).

Note (3.3) that nonnegativeness of $D N(u)$ (i.e. $D C(u)$ here) is not required in (3.4), (3.5) and (3.6).

4.7. LemmA. Suppose $\operatorname{dim} \operatorname{ker} D A_{\lambda}(u)=1$ and $\operatorname{ker} D A_{\lambda}(u)$ has generator $e$. Then the following properties are equivalent.

(i) $u$ is a fold point (3.1) of $A_{\lambda}$;

(ii) $(u, \lambda)$ is a fold point of $A$; 
(iii) $\langle C(e, e), C(e, u)\rangle_{H} \neq 0$; and

(iv) $\langle u, C e\rangle_{H} \neq 0$.

Proof. By definition (3.1), (i) is equivalent to $D^{2} A_{\lambda}(u)(e, e) \notin$ range $D A_{\lambda}(u)$, which by $(4.3)(\mathrm{v})$ is $\left\langle D^{2} A_{\lambda}(u)(e, e), e\right\rangle_{H} \neq 0$. The equivalence of (i) and (ii) is given in (3.4) (see the second paragraph of (3.3)). By (4.3)(ii), conclusion (i) is equivalent to

$$
0 \neq\left\langle D^{2} C(u)(e, e), e\right\rangle_{H}=4\langle C(e, C(e, u)), e\rangle_{H}+2\langle C(u, C(e, e)), e\rangle_{H},
$$

which by (4.2)(iii) is

$$
6\langle C(e, e), C(e, u)\rangle_{H}=6\langle u, C(e, C(e, e))\rangle_{H}=6\langle u, C e\rangle_{H},
$$

and the equivalence of (i), (iii) and (iv) results.

4.8. LEMMA. (i) Let $\operatorname{dim} \operatorname{ker} D A_{\lambda}(u)=1$ with $\operatorname{ker} D A_{\lambda}(u)$ generated by $e$; then $(u, \lambda)$ is a good point (3.1) of $A$ with $\omega=(0,1)$ if and only if $\langle L e, e\rangle_{H} \neq 0$.

(ii) If $\operatorname{dim} \operatorname{ker} D A_{\lambda}(0)=1$ with $\operatorname{ker} D A_{\lambda}(0)$ generated by $e$, then $\lambda \neq 0$ and $(0, \lambda)$ is a cusp point (3.1) of $A$ with $\omega=(0,1)$.

In case of compression only (4.5), $\langle L e, e\rangle_{H}>0$, so $u$ in (i) is necessarily a good point.

PROOF. From (4.3)(ii) and from the arguments of (3.5) with $a=\langle e, L u\rangle_{H}$,

$$
\left\langle D^{2} A(u, \lambda)((e, 0),(0,1)),(e, a)\right\rangle_{H \times \mathbf{R}}=-\langle L e, e\rangle_{H},
$$

yielding conclusion (i).

For (ii), since $0=D A_{\lambda}(0) \cdot e=e-\lambda L e+D C(0) \cdot e=e-\lambda L e$ by (4.6) and (1.2)(3)(c), $\lambda \neq 0$ and $e$ is an eigenvector of $v-\lambda L v=0$. Thus $\langle L e, e\rangle_{H} \neq 0$, so 0 is a good point of $A_{\lambda}$ by (i); from (4.7) 0 is not a fold point, so it is (3.1) a precusp point of $A_{\lambda}$ with $\omega=(0,1)$.

Now $(0, \lambda)$ is an (intrinsic) cusp point (3.1) (see the proof of (3.6)) if and only if

$$
m_{1}-m_{2} \neq 0,
$$

where

$$
\begin{aligned}
& m_{1}=\left\langle D^{3} A(0, \lambda)((e, 0),(e, 0),(e, 0)),(e, a)\right\rangle_{H \times \mathbf{R}}, \\
& m_{2}=3\left\langle D^{2} A(0, \lambda)((e, 0),(y, 0)),(e, a)\right\rangle_{H \times \mathbf{R}},
\end{aligned}
$$

$a=\langle e, L 0\rangle_{H}=0$ (proof of (3.4)) and

$$
y \in(D A(0, \lambda))^{-1}\left(D^{2} A(0, \lambda)((e, 0),(e, 0))\right) .
$$

From the proof of (3.4) and from (4.6) and (1.2)(3)(c),

$$
D^{2} A(0, \lambda)((e, 0),(e, 0))=\left(D^{2} C(0)(e, e), 0\right)=(0,0),
$$

so we may take $y=0$, and $m_{2}=0$. From (4.3)(iii) and (4.2)(i) and (ii), $m_{1}>0$, so that $m_{1}-m_{2} \neq 0$, and 0 is an intrinsic cusp point (3.1) and thus (3.2) a cusp point with $\omega=(0,1)$. 
4.9. THEOREM. (a) If $\lambda$ is not an eigenvalue of $v=\lambda L v$, then there are open neighborhoods $U$ and $V$ of $(0, \lambda)$ in $H \times \mathbf{R}$ such that $A: U \rightarrow V$ is a real analytic diffeomorphism (onto).

(b) If $\lambda_{j}$ is a simple eigenvalue of $v=\lambda L v$, then $\left(0, \lambda_{j}\right)$ is a cusp point (3.1) of $A$. Thus there are open neighborhoods $U$ and $V$ of $\left(0, \lambda_{j}\right)$ in $H \times \mathbf{R}$ and $C^{\infty}$ diffeomorphisms $\varphi$ and $\psi$ such that the diagram

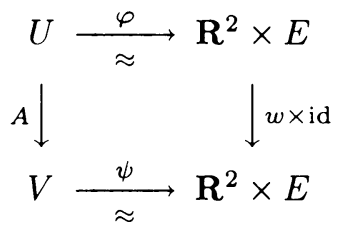

commutes, with $\varphi\left(0, \lambda_{j}\right)=(0,0,0)=\psi\left(0, \lambda_{j}\right)$. Here $E$ is the orthogonal complement of the eigenvector $v_{j}$ of $v=\lambda_{j} L v$, and $w(t, \lambda)=\left(t^{3}-\lambda t, \lambda\right)$.

ProOF. From (4.6) and $(1.2)(3)(\mathrm{c}) D C(0) \equiv 0$, so $D A_{\lambda}(0) \cdot v=0$ for $v \neq 0$ if and only if $\lambda$ is an eigenvalue of $v=\lambda L v$. Conclusion (a) results from (4.3)(v) and the Inverse Function Theorem [Z, p. 172].

Now assume the hypotheses of (b); then $\operatorname{dim} \operatorname{ker} D A_{\lambda_{j}}(0)=1$ and $e=v_{j}$ is a. generator of $\operatorname{ker} D A_{\lambda_{j}}(0)$. From (4.8)(ii), $\left(0, \lambda_{j}\right)$ is a cusp point of $A$ with $w=(0,1)$. The existence of the commuting diagram follows from (3.2).

4.10. REMARKS. If $\lambda_{m}$ is a simple eigenvalue, then in a neighborhood of $\left(0, \lambda_{m}\right)$ in $H \times \mathbf{R}\left[\mathbf{B F}-\mathbf{1}\right.$, Theorem 3, p. 1008] the number of solutions for $\lambda \leq \lambda_{m}$ is one (viz., $u \equiv 0$ ) and for $\lambda_{m}<\lambda$ is three (one of which is $u \equiv 0$ ). The given form [BF-1, Lemma 1, p. 1008] of the equations can be readily transformed into our form.

From the equivalence (4.9) of $w \times$ id and $A \mid U: U \rightarrow V$, the singular set $S(A \mid U)$ separates $U$ into two components $U_{1}$ and $U_{3}, A(S(A \mid U))$ separates $V$ into two components $V_{1}$ and $V_{3}, A$ maps $U_{3}$ diffeomorphically onto $V_{3}$, and for each $y \in V_{i}$, $(A \mid U)^{-1}(y)$ has $i$ points $(i=1,3)$. It follows that for $(0, \lambda) \in V, \lambda<\lambda_{m}$ if and only if $(0, \lambda) \in V_{1}$, and $\lambda_{m}<\lambda$ if and only if $(0, \lambda) \in V_{3}$.

The map $w \times$ id in (4.9) maps the singular set $S(w \times$ id) homeomorphically onto its image $(w \times \mathrm{id})(S(w \times \mathrm{id}))$, and each $(s, \lambda, v) \in(w \times \mathrm{id})(S(w \times \mathrm{id}))$ is either the image of a cusp point (if $(s, \lambda)=(0,0))$ or a fold point (if $(s, \lambda) \neq(0,0)$ ). For each $s \in \mathbf{R}$ and $v \in E$, the line $s \times \mathbf{R} \times v$ meets $(w \times \mathrm{id})(S(w \times \mathrm{id}))$ in precisely one point $p=p(s, v)$, and $(w \times \mathrm{id})^{-1}(s \times \mathbf{R} \times v)$ is (topologically) pictured in Figure 2(a) if $p$ is a cusp point image, and Figure 2(b) if $p$ is a fold point image, where the vertical direction is the $\lambda$ direction and the points indicated are $(w \times \mathrm{id})^{-1}(p)$. The map $A \mid U: U \rightarrow V$ is $C^{\infty}$ equivalent to $w \times$ id (4.9), and these diagrams are the bifurcation diagrams for $A$ given in [CR, pp. 140, 150, respectively], specifically, $2(\mathrm{a})$ is that for $A^{-1}(\delta g)$ where $\delta=0$ and $2(\mathrm{~b})$ is that for $0 \neq \delta \in R$ small and $g \in H$. Thus (4.9) provides a single map which unifies these various bifurcation diagrams.

4.11. REMARKS. If $\lambda_{1}>0, \lambda_{1}$ is simple, and $\lambda_{1}<\lambda<\lambda_{2}$, then the von Kármán equations have [AM, pp. 640 641] exactly three solutions.

In [CHM, pp. 179 181] the authors consider the von Kármán equations with an extra parameter $\mu$, viz., $A_{\lambda, \mu}(u, z)=u-\lambda L u+C(u)-\mu z$, for fixed $u, z \in H$. 


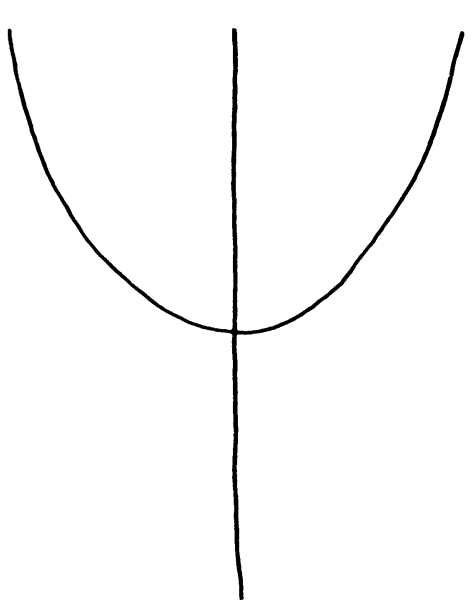

(a)

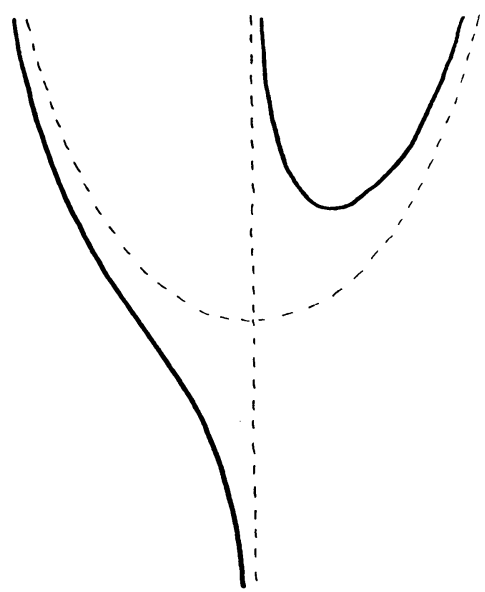

(b)

\section{FIGURE 2}

They study $A_{\lambda, \mu}(u, z)$ as a function of $(\lambda, \mu)$ and obtain a bifurcation curve with a cusp.

\section{REFERENCES}

[A] R. A. Adams, Sobolev spaces, Academic Press, New York, 1975.

[AM] A. Ambrosetti and G. Mancini, Sharp nonuniqueness results for some nonlinear problems, Nonlinear Anal. 3 (1979), 635-645.

[An] S. S. Antman, The influence of elasticity on analysis: Modern developments, Bull. Amer. Math. Soc. (N.S.) 9 (1983), 267-291.

[B-1] M. S. Berger, Nonlinearity and functional analysis, Academic Press, New York, 1977.

[B-2] _ _ Nonlinear problems with exactly three solutions, Indiana Univ. Math. J. 28 (1979), 689698.

[B-3] _ New applications of the calculus of variations in the large to nonlinear elasticity, Comm. Math. Phys. 35 (1974), 141-150.

[B-4] _ On von Kármán's Equations and the buckling of a thin elastic plate. I; The clamped plate, Comm. Pure Appl. Math. 20 (1967), 687-719.

[BC] M. S. Berger and P. T. Church, Complete integrability and perturbation of a nonlinear Dirichlet problem. I, Indiana Univ. Math. J. 28 (1979), 935-952. Erratum, ibid. 30 (1981), 799.

[BCT-1] M. S. Berger, P. T. Church and J. G. Timourian, An application of singularity theory to nonlinear elliptic partial differential equations, Singularities (P. Orlik, ed.), Proc. Sympos. Pure Math., vol. 40, Part 1, Amer. Math. Soc., Providence, R.I., 1983, pp. 119-126.

[BCT-2] _ Folds and cusps in Banach spaces, with applications to nonlinear partial differential equations. I, Indiana Univ. Math. J. 34 (1985), 1-19.

[BCT-3] _ Integrability of nonlinear differential equations via functional analysis, Nonlinear Functional Analysis and Its Applications (F. E. Browder, ed.), Proc. Sympos. Pure Math., vol. 45, Part 1, Amer. Math. Soc., Providence, R. I., 1986, pp. 117-123.

[BF-1] M. S. Berger and P. C. Fife, On von Kármán's equations and the buckling of a thin elastic plate, Bull. Amer. Math. Soc. 72 (1966), 1006-1011.

[BF-2] _ On von Kármán's equations and the buckling of a thin elastic plate. II: Plate with general boundary conditions, Comm. Pure Appl. Math. 12 (1968), 227-247.

[BJS] L. Bers, F. John, and M. Schecter, Partial differential equations, Wiley, New York, 1964.

$[\mathrm{CaC}]$ V. Cafagna and P. T. Church, in preparation. 
[CaD-1] V. Cafagna and F. Donato, Un résult global de multiplicité pour un problème différentiel nonlinéaire du premier ordre, C. R. Acad. Sci. Paris 300 (1985), 523-526.

[CaD-2] _ Singularity theory and the number of solutions to some nonlinear differential problems, preprint.

[CHM] S. Chow, J. K. Hale and J. Mallet-Paret, Applications of generic bifurcation. I, Arch. Rational Mech. Anal. 59 (1975), 159-188.

[CDT] P. T. Church, E. N. Dancer, and J. G. Timourian, in preparation.

[CT] P. T. Church and J. G. Timourian, The singular set of a nonlinear elliptic operator, Michigan Math. J. (to appear).

[CR] P. G. Ciarlet and P. Rabier, Les equations de von Kármán, Lecture Notes in Math., vol. 826, Springer-Verlag, New York, 1980.

[De] K. Deimling, Nonlinear functional analysis, Springer-Verlag, New York, 1985.

[Di] J. Dieudonné, Foundations of modern analysis, Academic Press, New York, 1960.

[GT] D. Gilbarg and N. S. Trudinger, Elliptic partial differential equations of second order, 2nd ed., Springer-Verlag, Berlin, 1983.

[GG] M. Golubitsky and V. Guillemin, Stable mappings and their singularities, Springer-Verlag, New York, 1973.

[HW] W. Hurewitz and H. Wallman, Dimension theory, Princeton Univ. Press, Princeton, N. J., 1941.

[K] V. L. Klee, Jr., A note on topological properties of normed linear spaces, Proc. Amer. Math. Soc. 7 (1956), 673-674.

[LM] F. Lazzeri and A. M. Micheletti, An application of singularity theory to nonlinear differentiable mappings between Banach spaces, preprint.

[M] W. S. Massey, Algebraic topology: An introduction, Springer-Verlag, New York, 1967; fourth corrected printing, 1977.

[Mc] H. P. McKean, Singularities of a simple elliptic operator, preprint.

[McS] H. P. McKean and J. C. Scovel, Geometry of some nonlinear operators, Ann. Pisa 12 (1986), 299-346.

[P] R. Palais, Natural operations on differential forms, Trans. Amer. Math. Soc. 92 (1959), 125141.

[R-1] B. Ruf, Multiplicity results for nonlinear elliptic equations, preprint.

[R-2] __ Singularity theory and the geometry of a nonlinear elliptic equation, preprint.

[Sc] J. T. Schwartz, Nonlinear functional analysis, Gordon and Breach, New York, 1969.

[St] J. J. Stoker, Nonlinear elasticity, Gordon and Breach, New York, 1968.

[Sz] A. Szulkin, On the number of solutions of some semilinear elliptic boundary value problems, Nonlinear Anal. 6 (1982), 95-116.

[Z] E. Zeidler, Nonlinear functional analysis. I. Fixed-point theorems, Springer-Verlag, New York, 1986.

Department of Mathematics, University of Massachusetts, Amherst, MASSACHUSETTS 01003

Department of Mathematics, Syracuse University, Syracuse, New York $13244-1150$

Department of Mathematics, University of Alberta, Edmonton, Alberta, CANADA T6G 2G1 